Iriarte-Pupo, A.J., Barreto Martínez, C.A., Campo-Landines, K. \& Domínguez De La Ossa, E. (2021). La inteligencia espiritual como estrategia para afrontar de manera constructiva el estrés laboral docente. Revista Electrónica Interuniversitaria de Formación del Profesorado, 24(1), 101-113.

DOI: https://doi.org/10.6018/reifop.435581

\title{
La inteligencia espiritual como estrategia para afrontar de manera constructiva el estrés laboral docente
}

\author{
Alberto Jesús Iriarte-Pupo ${ }^{1}$, Carmen Alicia Barreto Martínez², Kiara Campo-Landines², \\ Elsy Domínguez De La Ossa² \\ ${ }^{1}$ Universidad de Sucre, ${ }^{2}$ Universidad Tecnológica de Bolívar
}

\section{Resumen}

La actividad docente se ve amenazada frecuentemente por las condiciones precarias en las que se lleva a cabo. Los docentes están expuestos a diferentes riesgos de carácter psicosocial que afectan su desempeño y bienestar. El presente estudio pre-test post-test con grupo control tiene como objetivo examinar la incidencia de un programa de intervención basado en el desarrollo de la inteligencia espiritual (IE) sobre los niveles de estrés reportados por un grupo de 115 docentes de instituciones educativas oficiales de Sincelejo (Sucre, Colombia). Dentro de este programa de intervención, la IE se entiende como la capacidad de construir un sistema saludable (o adaptativo) de valores o creencias espirituales y de adoptarlo como estilo de vida. La IE abarca tres dimensiones fundamentalmente: cognitiva (conocimiento espiritual), afectiva (vivencia espiritual) y conductual (contingencia). Se hicieron análisis de tipo descriptivo e inferencial (intra-grupo e inter-grupo) y se compararon las variaciones de cada institución en relación con la percepción de los niveles de estrés de los docentes antes y después de la intervención (mediciones realizadas 2018 - 2019). Los resultados muestran que la percepción de los niveles de estrés de los docentes se transformaron significativamente, pasando de ser un factor de riesgo alto a uno bajo.

\section{Palabras clave}

Estrés laboral; docentes; inteligencia espiritual; factores psicosociales.

\section{Contacto:}

Alberto Jesús Iriarte Pupo, albertoiriarte4@yahoo.es. Universidad de Sucre. Cra 28 \# 5-267 Barrio Puerta Roja - Sincelejo.

Resultado parcial del proyecto Incidencia de un programa de inteligencia espiritual sobre la percepción de bienestar laboral docente en instituciones educativas públicas de Sincelejo-Sucre. 


\title{
Spiritual intelligence as a strategy for dealing constructively with teaching work stress
}

\begin{abstract}
Teachers' activity is frequently threatened by the precarious conditions in which this practice is carried out. Teachers are exposed to different psychosocial risks that affect their performance and well-being. The present pre-test post-test study with a control group aims to examine the incidence of a intervention program based on the development of spiritual intelligence (SI) about the levels of stress reported by a group of 115 teachers from official educational institutions in Sincelejo (Sucre, Colombia). Within this intervention program, SI is understood as the ability to build a healthy (or adaptive) system of spiritual values or beliefs and adopt it as a lifestyle. SI encompasses three fundamental dimensions: cognitive (spiritual knowledge), affective (spiritual experience) and behavioral (contingency). Descriptive and inferential analysis were performed (intragroup and intergroup) and the variations of each institution were compared with their relationship to the teachers' perception of the levels of stress before and after the intervention (measurements carried out on 2018 - 2019). The results show that teachers' perception of the levels of stress changed significantly from a high to a low risk factor.
\end{abstract}

\section{Key words}

Work stress; teachers; spiritual intelligence; psychosocial factors.

\section{Introducción}

La docencia es considerada como una de las profesiones de mayor impacto en el desarrollo humano, pues no solo incide en la transmisión y construcción de conocimientos, sino que también aporta a la construcción de las diferentes esferas de la sociedad. De manera tal que "las actitudes del docente, sus emociones, perspectivas, y expectativas se integran al proceso de enseñanza brindando un carácter particular a la experiencia áulica" (Carranco y Pando, 2019, p.525). Por tanto, el estudiante no solo recibe contenidos específicos, saberes estructurados, sino que también es formado en conocimientos volitivos y actitudinales que le sirven para afrontar diferentes áreas de la vida. Sin embargo, este proceso de enseñanzaaprendizaje e intercambio de saberes y experiencias se ve afectado porque los trabajadores de esta profesión están expuestos a diferentes riesgos, en esencial de carácter psicosocial, que amenazan su salud física y emocional, su bienestar y desarrollo y, en consecuencia, la forma en la que realizan su labor.

El Ministerio del Trabajo en Colombia (2016) señala que "los principales factores de riesgo psicosocial a los que están expuestos los educadores en Colombia varían según el sitio donde se desarrolle la labor, el ciclo académico y el tipo de institución (pública o privada)" (p. 9). Es decir, existe multiplicidad de condiciones que afectan la labor del docente, las cuales están determinadas por elementos contextuales, políticos y culturales, según el lugar donde los profesores se desempeñan. Asimismo, este Ministerio expresa que entre los factores de riesgo psicosocial más representativos en el sector educación se encuentran los siguientes: demandas ambientales y de esfuerzo físico, demandas emocionales, consistencia de rol, demandas cuantitativas, jornada de trabajo e influencia del ambiente laboral sobre el 
extralaboral, demandas de carga mental, capacitación y riesgo por problemas de orden público.

Diversas investigaciones indican que el estrés laboral es uno de los efectos en salud de los riesgos psicosociales a los que se encuentran expuestos los maestros al desempeñar su trabajo (Subaldo, 2012; Oramas, 2013; Parihuamán-Aniceto, 2017; Carranco y Pando, 2019). Así, se puede decir que los docentes sienten con cierta frecuencia los siguientes estados: cansancio físico y emocional, fuertes tensiones en cuanto a la solución de conflictos en aula, percepción de alta carga laboral, presiones por parte de los padres de familia, desosiego por el comportamiento descortés o irrespetuoso de los alumnos, exiguas perspectivas de promoción profesional, actitudes y comportamientos desfavorables de otros maestros (o de los directivos) en su contra, cantidad mayor de estudiantes en aula de lo esperado o regulado, imposición de altas expectativas por parte de la comunidad en general, apremio por la sujeción de una evaluación constante, entre otros. Tales problemáticas de orden organizacional e individual ponen de manifiesto la urgente necesidad de adelantar estrategias tendientes a intervenir o controlar las condiciones bajo las cuales los docentes realizan su trabajo y dotar a estos de habilidades y estrategias personales que les permitan afrontar o gestionar adaptativamente las múltiples demandas y exigencias que a menudo les propone el ambiente en el que se desenvuelven.

En este sentido, el objetivo particular de este escrito es dar a conocer la incidencia de un programa basado en el desarrollo de la inteligencia espiritual, sobre los niveles de estrés percibidos y reportados por los docentes. Asimismo, el tema investigado responde a las necesidades actuales colombianas, que desde el Ministerio de Educación Nacional se plantean, pretendiendo mejorar la calidad de vida de los maestros, por medio de diversas líneas estratégicas, tales como el planteamiento de "una política enfocada en la excelencia y el mejoramiento del ambiente laboral en los establecimientos educativos, partiendo del desarrollo del plan de acción de Bienestar y sus líneas de intervención” (MEN, 2016, p. 6).

\section{Algunos antecedentes investigativos}

Para Anandan y Paul (2017), la "inteligencia espiritual y el ajuste social son los dos constructos psicológicos que ayudan a cada individuo en el mundo moderno a llevar una vida mentalmente sana y orientada al valor" (p. 38). La investigación efectuada por los autores tuvo como conclusión que "la inteligencia espiritual y el ajuste social es un requisito urgente para el mundo de hoy. Por tanto, es responsabilidad de la autoridad escolar, los maestros y los padres, facilitar e inculcar valores con respecto a la Inteligencia Espiritual y el Ajuste Social" (p. 42).

Por su lado, la investigación realizada por Bhoslay (2015), titulada "A study of effectiveness of spiritual intelligence enhancement programme for trainee teachers" [Un estudio de la efectividad del programa de mejora de la inteligencia espiritual para maestros en formación], fue un recurso valioso para avanzar en la planeación y ejecución del programa basado en la inteligencia espiritual, que se puso en marcha con los maestros de Sincelejo. El objetivo principal de dicha investigación, fue "desarrollar un programa de mejora para optimizar la inteligencia espiritual de maestros en formación" (p. 1). Las competencias que se fortalecieron fueron las siguientes: pensamiento existencial crítico, producción de significado personal, conciencia trascendental y expansión del estado consciente. El investigador concluyó que el programa fue efectivo para desarrollar la inteligencia espiritual en los maestros en formación e incidió de manera positiva en las habilidades referentes al desarrollo de la inteligencia emocional, es decir, que les ayudó a tener mayor conciencia de sus emociones y sentimientos a la hora de tomar decisiones. 
Así también, Carranco y Pando (2019) ejecutaron un meta-análisis sobre los artículos existentes en cuanto a estrés laboral docente, vinculados al período 2013 - 2017. En este, los autores revisaron 31 artículos publicados en diversas revistas indexadas. En las investigaciones consultadas, se destacan aspectos relacionados con "las condiciones de trabajo, percepción del estrés laboral, autorealización, mobbing y estrés, estrés y satisfacción laboral, estrés laboral y actividad física, estrés laboral y salud mental, inteligencia emocional y estrés, estrés laboral y riesgo para la salud" (p. 538). En general, estos autores comentan que "los resultados obtenidos, ponen de manifiesto que hay un particular interés de la comunidad científica internacional por orientar sus esfuerzos investigativos alrededor del eje de la Salud Ocupacional y el Estrés Laboral” (p. 538). Asimismo, se reconoce que es necesario sostener un diagnóstico de los riesgos psicosociales a los que se ven expuestos los maestros. Finalmente, se confirma la necesidad de comprender la actividad magisterial como un proceso profesional de interés para el área de la salud, por el alto valor social que representa dicha labor, y el fuerte impacto que tiene sobre el desarrollo cultural de un contexto histórico particular.

\section{Sobre inteligencia espiritual}

El término inteligencia espiritual surgió de la clasificación de las diferentes tipologías de inteligencias categorizadas por Gardner (1998), quien propuso, entre otras, la inteligencia existencial, pero no profundizó en su conceptualización. Ahora bien, como lo expresa Gutiérrez (2015), Gardner define la inteligencia existencial como la capacidad para situarse a sí mismo con respecto a los rasgos existenciales de la condición humana como el significado de la vida, el significado de la muerte y el destino final del mundo físico y psicológico en profundas experiencias como el amor a otra persona o la inmersión en un trabajo de arte.

Por el contrario, la expresión inteligencia espiritual fue introducida por Zohar y Marshall (2001), quienes la conceptualizan como la capacidad de reformular y re-contextualizar la experiencia y, por ende, la capacidad para transformar la comprensión de la realidad. Para Zohar y Marshall (como se citó en Gutiérrez, 2015), la inteligencia espiritual es:

La inteligencia con la que afrontamos y resolvemos problemas de significado y valores, la inteligencia con la que podemos poner nuestros actos y nuestras vidas en un contexto más amplio, más rico y significativo, la inteligencia con la que podemos determinar que un curso de acción o un camino vital es más valioso que otro. (p. 45).

En síntesis, se puede determinar que la inteligencia espiritual es un sistema interno de creencias que produce el sentimiento de vivir con un sentido, estimula la esperanza, refuerza las normas sociales positivas y proporciona una red social de apoyo. Elementos que unidos entre sí mejoran el bienestar personal y, por lo tanto, las otras esferas de la vida, como la familiar y laboral. Teniendo en cuenta lo anteriormente expuesto, en el presente trabajo se entiende la inteligencia espiritual "como la capacidad de construir un sistema saludable (o adaptativo) de valores o creencias espirituales y de adoptarlo como estilo de vida" (Arias y Lemos, 2015, p. 96).

\section{Sobre estrés laboral docente}

La noción del término estrés es polisémica. Esta palabra no solo es usada frecuentemente por psicólogos, psiquiatras o sociólogos en su práctica profesional, sino también por las personas, en general, en la cotidianeidad. Además, es frecuente escucharla en los medios de comunicación masivos. El estrés es un fenómeno habitual en los diferentes entornos en los que se desenvuelve el ser humano, especialmente en el mundo laboral, en el que el individuo a menudo se ve sobrepasado o abrumado, debido a que percibe que sus capacidades no van 
acordes con las exigencias y demandas del ambiente, lo cual se traduce en reacciones o alteraciones fisiológicas y emocionales que afectan la salud.

Es posible definir el estrés como una reacción "inmediata y natural de nuestro organismo ante las situaciones y escenarios que nos resultan peligrosas, amenazadoras o desafiantes. En la actualidad, la vida y nuestro entorno cambiante nos exigen continuas adaptaciones; por tanto, cierta cantidad de estrés (activación) es necesaria" (Parihuamán-Aniceto, 2017, p. 27). Sin embargo, es importante señalar que las reacciones ante una situación estresante, dependen de la gravedad del evento, de los recursos personales con los que cuenta el individuo incluyendo el apoyo social y familiar (Díazb, 2011). Esta autora señala que, de acuerdo con el Subcentro de Seguridad Social y Riesgos Profesionales, el estrés puede definirse mejor como el conjunto de respuestas de carácter psicológico, emocional y conductual que se genera cuando el individuo enfrenta situaciones derivadas de su interacción con el medio y ante las cuales valora que su capacidad de afrontamiento resulta insuficiente, lo cual produce al mismo tiempo un desbalance que afecta su salud.

Con respecto a los docentes, como ya fue planteado, el estrés laboral puede ser causado por multiplicidad de factores y sus consecuencias alcanzan a repercutir en su desempeño diario, lo cual afecta a su vez el trato con sus estudiantes, con los padres de familia, y sus compañeros de trabajo.

\section{Metodología}

\section{Participantes}

Los grupos objetos de estudio fueron conformados por medio de una técnica de muestreo no probabilístico por conveniencia que según Otzen y Manterola (2017): "permite seleccionar aquellos casos accesibles que acepten ser incluidos (p.230). Además, el diseño seleccionado fue de tipo cuasiexperimental con grupo control (Hernández, Fernández y Baptista, 2014). Esto se realizó con el propósito de establecer la incidencia de una variable independiente (inteligencia espiritual) en otra dependiente (estrés laboral percibido).

En consecuencia, se tuvo como universo dos instituciones educativas de carácter oficial, pertenecientes al municipio de Sincelejo-Sucre (Colombia), ambas con condiciones similares y ubicadas en la zona urbana. La primera (Institución Educativa $\mathrm{N}^{\circ} 1$ ) cuenta con 57 docentes, discriminados de la siguiente manera: 38 mujeres y 19 hombres. La segunda (Institución Educativa $\mathrm{N}^{\circ} 2$ ) está conformada por 58 docentes, distribuidos así: 36 mujeres y 22 hombres. En ambos casos los docentes laboran en diferentes niveles educativos, que van desde el preescolar (estudiantes en edades entre 5 y 6 años) hasta la educación media (estudiantes con edades entre 15 y 17 años). Se escogió como grupo experimental de la población total de docentes, aquellos pertenecientes a la Institución Educativa $\mathrm{N}^{\circ} 1$, para ser intervenidos con el programa, dado que al aplicar por primera vez la batería para la evaluación de factores de riesgo psicosocial, se encontró que los funcionarios reportaron más condiciones de riesgo que aquellos de la Institución Educativa $\mathrm{N}^{\circ} 2$ (grupo control).

\section{Instrumento de recolección de datos}

Con el fin de medir la percepción sobre síntomas asociados al estrés que tienen los docentes, se decidió trabajar con el "Cuestionario para la evaluación del estrés (tercera versión)" que se encuentra en la "Batería de instrumentos para la evaluación de factores de riesgo psicosocial” (Ministerio de la Protección Social, 2010). Este cuestionario "es un instrumento diseñado para evaluar síntomas reveladores de la presencia de reacciones de estrés, distribuidos en cuatro categorías principales según el tipo de síntomas de estrés: a) 
fisiológicos, b) comportamiento social c) intelectuales y laborales y d) psicoemocionales" ( $p$. 371). El cuestionario consta de 31 ítems, distribuidos entre los diferentes síntomas expuestos anteriormente.

Es importante aclarar, que el cuestionario cuenta con un alto grado de consistencia interna, validez (realizada por medio del juicio de expertos) y confiabilidad (alfa de Cronbach entre $0,81$ y 0,90$)$.

\section{Procedimiento}

Después de aplicar el cuestionario para la evaluación de estrés, se diseñó un programa de intervención de acuerdo con lo propuesto por Arias y Lemos (2015), quienes operacionalizan el concepto de inteligencia espiritual desde tres áreas o dimensiones fundamentalmente: cognitiva (conocimiento espiritual); afectiva (vivencia espiritual) y conductual (contingencia). Desde estas dimensiones se trabajaron dieciocho aspectos, conformando agrupaciones de seis para cada dimensión. Entre estos aspectos se destacan: razonamiento moral, búsqueda de sentido, autoconocimiento, autotrascendencia, admiración por lo misterioso, paz interior, control de los impulsos y el ejercicio de la solidaridad. Después de realizar la intervención con el programa de inteligencia espiritual, se hizo nuevamente la evaluación para conocer los niveles de estrés reportados por los docentes.

En la propuesta de intervención, que tuvo una duración de cinco meses (inició en octubre de 2018 y culminó en marzo de 2019), se buscó articular las tres dimensiones de la inteligencia espiritual en los seminarios-talleres realizados con los docentes. Estos tuvieron una duración estimada de tres horas presenciales y dos horas de ejercicios prácticos y autónomos y se llevaron a cabo cada 15 días. Por ejemplo, en el seminario-taller $\mathrm{N}^{\circ} 2$, se realizó el trabajo articulando las facetas de la autotrascendencia, la empatía y el ejercicio de la solidaridad, las cuales hacen parte del grupo $\mathrm{N}^{\circ} 4$ de la tabla 1. De esta manera, en cada seminario-taller se pretendió brindar herramientas a los participantes, que permitieran desarrollar en ellos diferentes habilidades que hacen parte de la inteligencia espiritual.

Tabla 1.

Articulación de las distintas dimensiones y sus aspectos.

\begin{tabular}{cccc}
\hline $\begin{array}{l}\text { Grupos } \\
\text { articulados }\end{array}$ & Dimensión cognitiva & Dimensión afectiva & Dimensión conductual \\
\hline 1 & Razonamiento moral & Admiración por lo misterioso & Sobriedad, sencillez \\
2 & Práctica de la meditación & Paz interior & Control de los impulsos \\
3 & Autoconocimiento & Felicidad & Cuidado de la salud \\
4 & Autotrascendencia & Empatía & Ejercicio de la solidaridad \\
5 & Actitud frente al dolor & Entusiasmo & Estoicismo o resistencia física \\
6 & Búsqueda de sentido & Sensibilidad estética & Práctica del amor \\
\hline \multicolumn{2}{c}{ Elaboración propia teniendo en cuenta los aspectos de cada dimensión de la inteligencia espiritual. }
\end{tabular}

El análisis de los datos se realizó a través del Software SPSS versión 20.0, de acuerdo a tres procesos diferentes a saber: descriptivo por cada grupo participante, inferencial (intragrupo) en el que se comparan las variaciones de cada institución al transcurrir el tiempo con respecto a las dos mediciones realizadas (2018 - 2019) e inferencial (intergrupo) en el que se contrastan las variaciones entre los grupos participantes antes y después de que el grupo experimental fuera intervenido. 


\section{Resultados}

En este apartado se presentan los resultados del análisis de los datos recolectados en diferentes momentos del proceso investigativo. Primero se muestran los resultados de los estadísticos que describen algunas características sociodemográficas de los sujetos investigados. Luego, se exponen los resultados obtenidos de la comparación intragrupos y entre grupos, mediante pruebas no paramétricas, con el fin de determinar la incidencia de la variable independiente (programa basado en la teoría de la inteligencia espiritual) en la dependiente (percepción sobre los síntomas del estrés).

\section{Equivalencia inicial entre los grupos control y experimental: control de variables}

Con el fin de comprobar que los grupos eran inicialmente equiparables, en una serie de variables de control, se ha puesto a prueba la igualdad por medio de pruebas no paramétricas, las cuales no se basan en la suposición de normalidad de la distribución de probabilidad a partir de las que fueron obtenidos los datos. La prueba no paramétrica utilizada para la comparación de variables fue la prueba $U$ de Mann-Whitney. En las siguientes tablas, se muestra que no existen diferencias significativas en cuanto a las variables edad, sexo, nivel de escolaridad y estrato social de los participantes.

Tabla 2.

Resultados de la prueba U de Mann-Whitney para variables de control.

\begin{tabular}{lllccccc}
\hline \multicolumn{1}{c}{$\begin{array}{c}\text { Variables de } \\
\text { control }\end{array}$} & \multicolumn{1}{c}{ Grupos } & N & $\begin{array}{c}\text { Rango } \\
\text { promedio }\end{array}$ & $\begin{array}{c}\text { Suma de } \\
\text { rangos }\end{array}$ & $\begin{array}{c}\text { U de } \\
\text { Mann- } \\
\text { Whitney }\end{array}$ & Z & $\begin{array}{c}\text { Sig. } \\
\text { Asintótica } \\
\text { (bilateral) }\end{array}$ \\
\hline \multirow{2}{*}{ Rango de edad } & Control & 42 & 37,38 & 1570,00 & 635,000 & $-0,191$ & 0,848 \\
& Experimental & 31 & 36,48 & 1131,00 & & 0,924 \\
Sexo & Control & 42 & 37,17 & 1561,00 & 644,000 & $-0,096$ & 0,764 \\
& Experimental & 31 & 36,77 & 1140,00 & & & \\
Nivel de escolaridad & Control & 42 & 37,55 & 1577,00 & 628,000 & $-0,301$ & 0,245 \\
\multirow{2}{*}{$\begin{array}{l}\text { Estrato social } \\
\text { vivienda }\end{array}$} & Experimental & 31 & 36,26 & 1124,00 & & & \\
\hline
\end{tabular}

Elaboración propia utilizando el programa SPSS versión 20.0.

\section{Control de algunas variables de tipo cualitativas}

Con respecto a este tipo de variables, se puede decir que las dos instituciones educativas son de carácter oficial, atienden entre 800 y 1200 estudiantes, su infraestructura es propia y la cantidad de docentes y directivos docentes con los que cuentan son similares (entre 40 y 50 ). Asimismo, se puede decir que las condiciones ambientales son parecidas, debido a que ambas se encuentran en la zona urbana de la cabecera municipal. Además, el instrumento utilizado para el proceso investigativo es el mismo para ambos grupos y se aplica al mismo tiempo (con uno o dos días de diferencia).

\section{Estadísticos descriptivos de los niveles de estrés percibidos por los docentes}

En el siguiente gráfico se da cuenta comparativamente de la percepción que tienen los docentes sobre los niveles de estrés que están manejando. El pre-test fue realizado en el mes de julio del año 2018 y el pos-test fue realizado en el mismo mes del año 2019, ya que la batería 
de riesgos psicosociales se debe aplicar por lo menos con un año de diferencia según la Resolución 2646 del 2008 (Ministerio de la Protección Social, 2010).

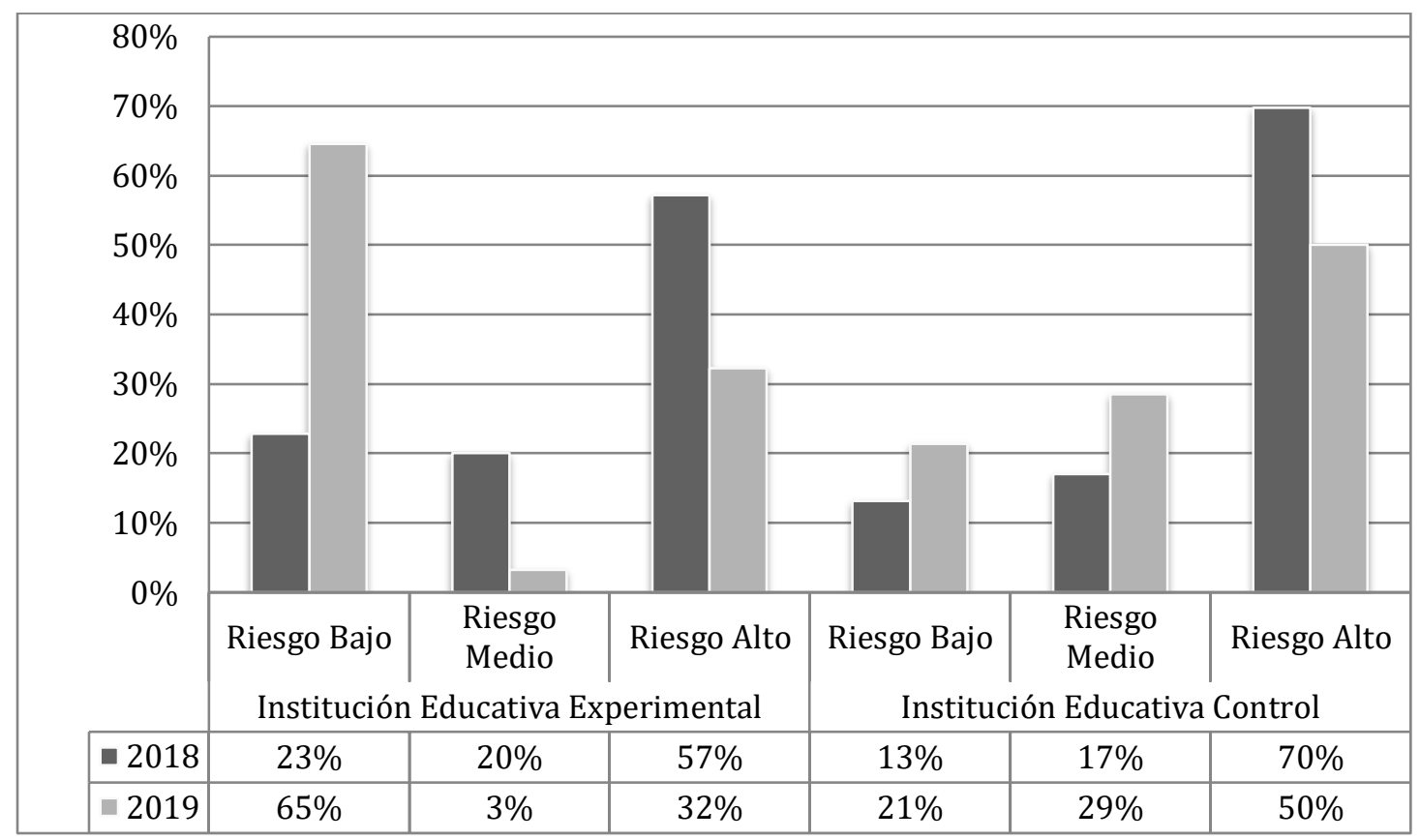

Figura 1. Gráfico comparativo percepción de estrés años 2018 y 2019 entre instituciones.

Con respecto a los niveles de estrés manejados por docentes y directivos docentes en la Institución Educativa $\mathrm{N}^{\circ} 2$ (grupo control) durante el año 2018; se logra evidenciar en la figura 1 que el $70 \%$ de los encuestados perciben que tienen un nivel de riesgo alto. Esto sugiere que ellos pueden presentar varios síntomas físicos y emocionales o psicológicos asociados al estrés. Asimismo, se puede observar que un $57 \%$ de los docentes y directivos docentes de la Institución Educativa $\mathrm{N}^{\circ} 1$ (grupo experimental) perciben que tienen el mismo nivel de riesgo de estrés. Con respecto al mismo año, solo un $23 \%$ y un $13 \%$ de los docentes y directivos docentes de ambas instituciones, respectivamente, perciben que tienen un nivel de riesgo bajo.

Por su parte, en el año 2019, luego de la intervención realizada con el programa; se evidencia que la percepción del nivel de riesgo alto, en la Institución Educativa $\mathrm{N}^{\circ} 2$ (grupo control), descendió 20 puntos porcentuales, de los cuales 12 pasaron al nivel de riesgo medio y 8 al nivel de riesgo bajo. Con respecto a la Institución Educativa $\mathrm{N}^{\circ} 1$ (grupo experimental), se aprecia el descenso de 15 puntos porcentuales del nivel de riesgo alto y de 17 del nivel de riesgo medio. De igual forma, la percepción del nivel de riesgo bajo pasó de $23 \%$ a un $65 \%$, lo cual muestra un cambio representativo de más de 40 puntos porcentuales.

\section{Resultados comparativos intra-grupos (2018-2019)}

En este apartado se presenta, si existieron o no, diferencias estadísticamente significativas en la percepción de los docentes en cuanto a la variable estrés, después de un año de haber aplicado la batería para la evaluación de riesgos psicosociales en ambas instituciones. Por lo tanto, se realizó una prueba de signos bilateral para dos muestras relacionadas con el fin de efectuar las comparaciones. Esta prueba permite contrastar la hipótesis de que las respuestas a dos tratamientos pertenecen a poblaciones idénticas y requiere que las respuestas estén medidas por lo menos en una escala ordinal. Al contrastar si hubo o no 
diferencias significativas al pasar el tiempo en el grupo control, con respecto a los niveles de estrés, la prueba de signos arrojó el siguiente resultado:

Tabla 3.

Prueba de signos comparación año 2018 y 2019 niveles de estrés, grupo control.

\begin{tabular}{lc}
\hline \multicolumn{2}{c}{ Estadísticos de prueba - Prueba de signos } \\
\hline & $\begin{array}{l}\text { Niveles de estrés } 2019 \text { - Niveles } \\
\text { de estrés } 2018\end{array}$ \\
$\mathrm{Z}$ & $-1,107$ \\
$\begin{array}{l}\text { Sig. Asintótica } \\
\text { (bilateral) }\end{array}$ \\
$\begin{array}{l}\text { Elaboración propia utilizando el programa SPSS versión } \\
20.0\end{array}$
\end{tabular}

Como se observa, el $p$-valor $(0,268)$ mayor a 0,05 indica que se acepta la hipótesis nula. Es decir, que no existen diferencias significativas con respecto a la percepción de los niveles de estrés manejados por los docentes del grupo control. Ahora bien, a pesar de no haber diferencias significativas, sí se reconoce que la percepción sobre el estrés que tienen los encuestados pasó de tener un riesgo alto a un riesgo medio, lo que puede indicar que las directivas institucionales llevaron a cabo algunas intervenciones, después de haber recibido el informe, con el fin de mejorar la situación antes descrita. Así, al efectuar la misma prueba en el grupo experimental en el cual se realizó la intervención, esta arrojó los siguientes datos con respecto a los niveles de estrés:

Tabla 4.

Prueba de signos comparación año 2018 y 2019 niveles de estrés, grupo experimental.

\begin{tabular}{l}
\hline \multicolumn{2}{c}{ Estadísticos de prueba - Prueba de signos } \\
\hline \\
$\begin{array}{l}\text { Niveles de estrés } 2019 \text { - Niveles } \\
\text { de estrés } 2018\end{array}$ \\
$\mathrm{Z}$ \\
$\begin{array}{l}\text { Sig. Asintótica } \\
\text { (bilateral) }\end{array}$ \\
$\begin{array}{l}\text { Elaboración propia utilizando el programa SPSS versión } \\
\text { 20.0. }\end{array}$
\end{tabular}

Los resultados de la prueba de signos ( $p$-valor 0,001) muestran que sí existen diferencias significativas en las percepciones de los docentes, en cuanto a los niveles de estrés que ellos reportaron, medidos en los diferentes tiempos. Este es un buen indicador sobre la influencia del programa de intervención basado en la teoría de la inteligencia espiritual. En otras palabras, el programa diseñado incidió positivamente en la disminución de los niveles de estrés de los docentes participantes, al ofrecer mejores estrategias para afrontar las condiciones adversas. 


\section{Resultados comparativos entre-grupos (2018-2019)}

En este apartado se expone, si existieron o no, diferencias estadísticamente significativas en cada uno de los factores que evalúa la encuesta sobre los niveles de estrés, al cotejar ambas instituciones (grupo experimental con el control). Se usó, entonces, la prueba U de MannWhitney para hacer las comparaciones entre-grupos y establecer si se dieron o no diferencias significativas entre estos. Esta prueba es paralela a la prueba paramétrica de contraste $t$ de student para muestras independientes, con la cual se contrasta si dos poblaciones muestreadas son equivalentes en su posición, con el fin de evaluar si hubo o no efectos sobre la variable dependiente. En este sentido, la prueba aplicada dio como resultado lo siguiente:

Tabla 5.

Prueba de Mann-Whitney comparación niveles de estrés grupo control y experimental año 2018 y 2019.

\begin{tabular}{llccc}
\hline \multicolumn{5}{c}{ Rangos } \\
\hline \multirow{4}{*}{ Niveles de estrés 2018 } & \multicolumn{1}{c}{ Grupo } & $\mathrm{N}$ & Rango promedio & Suma de rangos \\
& Control & 42 & 37,64 & 1581,00 \\
& Experimental & 31 & 36,13 & 1120,00 \\
& Total & 73 & & \\
Niveles de estrés 2019 & Control & 42 & 42,79 & 1797,00 \\
& Experimental & 31 & 29,16 & 904,00 \\
& Total & 73 & & \\
\hline
\end{tabular}

\begin{tabular}{lrr}
\hline \multicolumn{2}{c}{ Estadísticos de contraste - Variable de agrupación: Grupo } \\
\hline & $\begin{array}{c}\text { Niveles de estrés } \\
2018\end{array}$ & $\begin{array}{r}\text { Niveles de estrés } \\
\text { U de Mann-Whitney }\end{array}$ \\
W de Wilcoxon & 624,000 & 408,000 \\
Z & 1120,000 & 904,000 \\
$\begin{array}{l}\text { Sig. Asintótica } \\
\text { (bilateral) }\end{array}$ & $-0,301$ & $-2,712$ \\
\hline
\end{tabular}

Elaboración propia utilizando el programa SPSS versión 20.0.

En la tabla 5, en cuanto al año 2018, se evidencia que el rango promedio de los niveles de estrés manejados por los profesores de ambas instituciones era muy similar. Es decir, la prueba estadística arroja que no existen diferencias significativas entre ambos grupos ( $\mathrm{p}$ valor 0,763 ). En cuanto al año 2019, si bien el rango promedio de la percepción sobre el nivel de estrés del grupo control disminuyó, se nota que el rango promedio de nivel de estrés del grupo experimental bajó considerablemente. Esto se ve reflejado en un p-valor menor a 0,05. Lo anterior quiere decir que sí hubo diferencias estadísticamente significativas ( $p$-valor 0,007 ), en relación con los procesos de manejo de estrés que brindó el programa de intervención basado en la teoría de la inteligencia espiritual, para el grupo intervenido.

Los diagramas de caja dan muestra de lo ocurrido antes y después del proceso interventivo. Se aprecia que, en el año 2019, los maestros pertenecientes a la institución educativa del grupo experimental bajaron notablemente la puntuación sobre la percepción que tenían de sus niveles de estrés. 

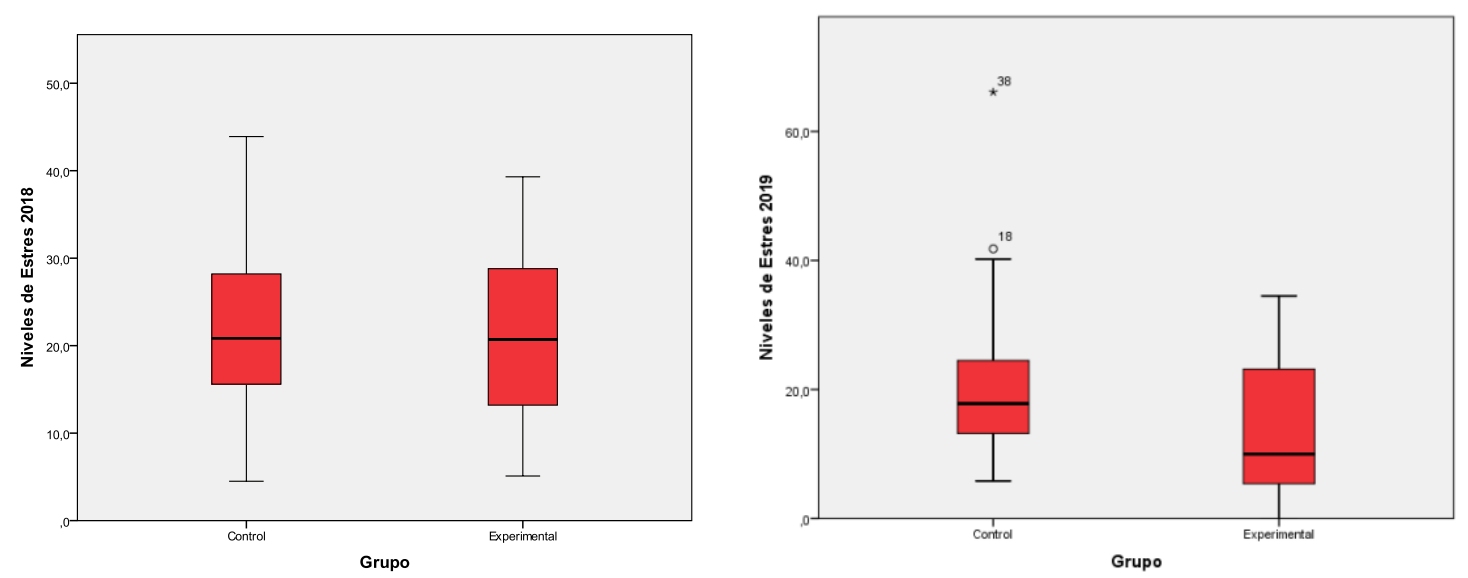

Figura 2. Diagramas de caja comparativos año 2018 y 2019, de los niveles de estrés grupo control y experimental.

\section{Discusión y conclusiones}

Los resultados analizados anteriormente muestran, con respecto al grupo experimental, que la percepción sobre los niveles de estrés de los profesores y directivos docentes se transformó significativamente, pasando de ser un factor de riesgo alto a ser uno de riesgo bajo. Consecuentemente, los aspectos investigativos corroboran que el desarrollo de la inteligencia espiritual responde a las siguientes necesidades de los seres humanos: vivir una vida con sentido, con significado; buscar la reconciliación consigo mismo, con la vida, los demás y el universo; así como buscar la verdad, la libertad plena y verdadera, la soledad y el silencio ocasional, y un orden en la vida (Torralba, 2010). Lo anterior se refleja en la percepción sobre los bajos niveles de estrés que manejan los docentes y directivos docentes, luego de conocer y llevar a cabo el programa basado en la inteligencia espiritual.

Asimismo, se corrobora lo planteado por Singh \& Sinha (2013), quienes plantearon que la inteligencia espiritual impacta positivamente en la calidad de vida. Por lo tanto, es imperativo que los seres humanos lleven una vida plena y satisfactoria mediante la mejora de este tipo de inteligencia, porque es un factor fundamental para lograr tal objetivo. A su vez, estos autores recalcan que el cerebro está conectado con la inteligencia espiritual, pero permanece inactivo y necesita ser activado, lo cual se intentó realizar mediante la puesta en marcha del programa.

Hay que tener en cuenta, además, que el diseño del programa estuvo basado en las recomendaciones que algunos autores hacen con respecto a la necesidad de usar métodos que ayudan a desarrollar la inteligencia espiritual, tales como los siguientes: la meditación, la oración, las plegarias o los mantras; al igual que herramientas de empoderamiento, como por ejemplo: el Reiki y la observación permanente de valores/cualidades espirituales. Si se realizan estos ejercicios de manera diaria se logra "a positive virtuous spiral" [una espiral virtuosa positiva] (Singh \& Sinha, 2013, p.5). También es importante señalar que las condiciones individuales, culturales, sociales, así como las percepciones, opiniones, creencias, valores, etc. influyen en las reacciones emocionales, psicológicas y conductuales de los sujetos frente a situaciones amenazantes o complejas.

Mediante lo anteriormente expuesto, se reconoce y comprueba a nivel experimental lo planteado por Zohar y Marshall (2001); puesto que el programa de intervención demostró que poner en práctica diferentes actividades que coadyuven a mejorar esta capacidad de afrontamiento de los problemas, incide de manera positiva en el desempeño laboral y vital de las personas. En síntesis, se puede decir que la inteligencia espiritual, entendida como un 
sistema interno de creencias, produce un sentimiento de vivir con un sentido, estimula la esperanza, refuerza las normas sociales positivas y proporciona una red social de apoyo. Elementos que unidos entre sí mejoran el bienestar personal y, por consiguiente, las otras esferas de la vida, como la familiar y laboral.

\section{Referencias}

Anandan, K. \& Paul, G. (2017). A study on spiritual intelligence among higher secondary students in relation to their social adjustment. Journal of Research in Humanities and Social Science, 5(3), 38-42. ISSN (Online): 2321-9467. Recuperado de http://www.questjournals.org/jrhss/papers/vol5-issue3/F533842.pdf

Arias, R. y Lemos, V. (2015). Una aproximación teórica y empírica al constructo de inteligencia espiritual. Revista Enfoques, XXVII (1), 79-102. Recuperado de http://uap.edu.ar/wpcontent/uploads/2015/11/2015-1-Lemos-Arias.pdf

Bhoslay, R. (2015). A study of effectiveness of spiritual intelligence enhancement programme for trainee teachers. International Conference on Contemporary Issues in Education, 18. Dubai - United Arab Emirates. Recuperado de http://iccieconference.net/2015/pdf/951\%20-\%20Raamaa\%20\%20Bhoslay.pdf

Carranco, S. y Pando, M. (2019). Metanálisis de los artículos sobre estrés laboral docente en el período 2013 - 2017. Revista Científica Mundo de la Investigación y el Conocimiento, $3(1), 522-554$.

Díaz, D.L. (2011). Estrés laboral y sus factores de riesgo psicosocial. Revista CES Salud Pública, 2(1), 80-84. ISSN 2145-9932. Recuperado de http://revistas.ces.edu.co/index.php/ces_salud_publica/article/download/1451/914

Gardner, H. (1998). Inteligencias múltiples.Teoría en la práctica. México: Editorial Paidós.

Gutiérrez, M. (2015). Estado del arte de la teoría de la inteligencia espiritual (Tesis de maestría). Universidad Icesi, Santiago de Cali, Colombia.

Hernández, R., Fernández, C. y Baptista, P. (2014). Metodología de la investigación (6a. ed.). México: McGraw-Hill.

Ministerio de Educación Nacional. (2016). Política integral de excelencia docente \& bienestar Laboral: Calidad de Vida en la escuela. Recuperado de http://www.sednortedesantander.gov.co/sitio/images/documentos/bienestarlaboral /Politica\%20Excelencia\%20Docente\%20y\%20Bienestar\%20Laboral.pdf

Ministerio de la Protección Social. (2010). Batería de instrumentos para la evaluación de factores de riesgo psicosocial. Recuperado de https://portal.posipedia.co/wpcontent/uploads/2019/08/bateria-instrumento-evaluacion-factores-riesgopsicosocial.pdf

Ministerio del Trabajo-Colombia. (2016). Prácticas de trabajo saludable para educadores. Protocolo de intervención de factores psicosociales en el sector educativo. Recuperado de http://fondoriesgoslaborales.gov.co/wp-content/uploads/2018/09/07-Protocolointervencion-sector-educativo.pdf

Oramas, A. (2013). Estrés laboral y síndrome de burnout en docentes cubanos de enseñanza primaria (Tesis doctoral). Escuela Nacional de Salud Pública: La Habana-Cuba: 
Otzen, T. y Manterola, C. (2017). Técnicas de muestreo sobre una población a Estudio. Int. J. Morphol., $\quad 35(1), \quad 227-232$. http://www.scielo.cl/pdf/ijmorphol/v35n1/art37.pdf

Parihuamán-Aniceto, M. (2017). Nivel de estrés de los docentes de las instituciones educativas de Villa Vicús y kilómetro 50, distrito de Chulucanas - Morropón - Piura (Tesis de maestría). Universidad de Piura, Piura, Perú.

Singh, MP. \& Sinha, J. (2013). Impact of spiritual intelligence on quality of life. International Journal of Scientific and Research Publications, 3(5), 1-5. ISSN 2250-3153. Recuperado de http://www.ijsrp.org/research-paper-0513/ijsrp-p1705.pdf

Subaldo, L. (2012). Las repercusiones del desempeño docente en la satisfacción y desgaste del profesorado (Tesis de maestría). Universidad de Valencia, Valencia, España.

Torralba, F. (2010). Inteligencia espiritual. Barcelona, España: Plataforma Actual.

Zohar, D. y Marshall, I. (2001). Inteligencia espiritual. Madrid, España: Plaza y Janés. 